\title{
Stable IIR Notch Filter Design with Optimal Pole Placement
}

\author{
Chien-Cheng Tseng, Senior Member, IEEE, and Soo-Chang Pei, Fellow, IEEE
}

\begin{abstract}
This paper presents a two-stage approach for designing an infinite impulse response (IIR) notch filter. First, the numerator of the transfer function of the IIR notch filter is obtained by placing the zeros at the prescribed notch frequencies. Then, the denominator polynomial is determined by using an iterative scheme in which the optimal pole placements are found by solving a standard quadratic programming problem. For stability, the pole radius in the single notch filter design is specified by the designer, and in the multiple notch filter design, the pole radius is constrained by using the implications of Rouché's theorem. Examples are included to illustrate the effectiveness of the proposed techniques.
\end{abstract}

Index Terms-Notch filters, quadratic programming.

\section{INTRODUCTION}

$\mathbf{N}$ OTCH filters have been an effective means for eliminating narrowband or sinusoidal interferences in certain signal processing applications ranging from power line interference cancelation for electrocardiograms to multiple sinusoidal interference removal for corrupted images. For the one-dimensional (1-D) case, several methods for the design and performance analysis of IIR and FIR notch filters have been developed; see [1]-[7], among others. For the two-dimensional (2-D) case, Pei and co-workers [8] and [9] proposed the methods that reduce the design of a stable IIR 2-D notch filter to the designs of several simple 1-D IIR filters. Usually, the IIR notch filter is preferred when notch bandwidth is very small because it requires less computation load than FIR notch filter.

During the past two decades, the IIR notch filters have been designed by algebraic methods without performing any optimization procedure. The well-documented methods can be divided into the following two groups. The first is that the zeros of IIR notch filter are constrained to lie on the unit circle whose angles are equal to notch frequencies, and the poles are placed at the same radial line as zeros. A typical pole-zero diagram and frequency response of this kind of filter are shown in Fig. 1. Thus far, these types of IIR filters have been widely used in the adaptive notch filtering applications to estimate and track the unknown frequencies of sinusoidal signals. The second is notch filter $H(z)$ synthesized by using the allpass filter $F(z)$ since both filters have the relation $H(z)=1 / 2(1+F(z))$. In this type of notch filter, the zeros also lie on the unit circle

Manuscript received June 14, 1999; revised August 14, 2001. The associate editor coordinating the review of this paper and approving it for publication was Dr. Xiang-Gen Xia.

C.-C. Tseng is with the Department of Computer and Communication Engineering, National Kaohsiung First University of Science and Technology, Kaohsiung, Taiwan, R.O.C. (e-mail: tcc@ccms.nkfust.edu.tw).

S.-C. Pei is with the Department of Electrical Engineering, National Taiwan University, Taipei, Taiwan, R.O.C.

Publisher Item Identifier S 1053-587X(01)09601-5. with angles equal to notch frequencies, but the poles are only near the zeros and do not have the same radian angles as zeros. Fig. 2 depicts the pole-zero diagram and frequency response of this type of notch filter. The main advantage of this method is that the mirror-image symmetry relation between numerator and denominator polynomials of $F(z)$ can be exploited to obtain a computationally efficient lattice filter realization with very low sensitivity.

From the conventional IIR notch filters, we see that the IIR notch filters can be designed by a two-stage approach. First, place the zeros on the unit circle with angles equal to notch frequencies such that the frequency response of filter has zero gains at the notch frequencies. Second, place the poles inside the unit circle and near zeros to the make frequency response of the non-notch band be a unit gain. Now, a question may be asked. What is the optimal pole placement? The pole placements of conventional methods are just two subjective choices. In this paper, the denominator polynomial of the transfer function of the IIR notch filter is determined by using an iterative scheme in which the optimal pole placements are found by solving a standard quadratic programming problem. For stability, the pole radius in the single notch filter design is specified by the designer, and in the multiple notch filter design, the pole radius is constrained by using the implications of Rouché's theorem. Examples are included to illustrate the proposed techniques. As a result, our design method has smaller errors than the conventional methods.

\section{Problem Statement}

The frequency response specification for ideal notch filter is given by

$$
D(\omega)= \begin{cases}0, & \omega= \pm \omega_{N k}, \\ 1, & \text { otherwise }\end{cases}
$$

where $\omega_{N k}$ are the notch frequencies. Without loss of generality, we assume that $\omega_{N 1}<\omega_{N 2}<\cdots<\omega_{N M}$. During the past two decades, the notch filters have been designed by algebraic methods without using any optimization procedures [10]-[13]. The well-established methods can be divided into the following two types:

\section{A. Type I}

The transfer function of this type notch filter is given by

$$
\begin{aligned}
H_{1}(z) & =\frac{\prod_{k=1}^{M} 1-2 \cos \left(\omega_{N k}\right) z^{-1}+z^{-2}}{\prod_{k=1}^{M} 1-2 r \cos \left(\omega_{N k}\right) z^{-1}+r^{2} z^{-2}} \\
& =\frac{B(z)}{B\left(r^{-1} z\right)}
\end{aligned}
$$



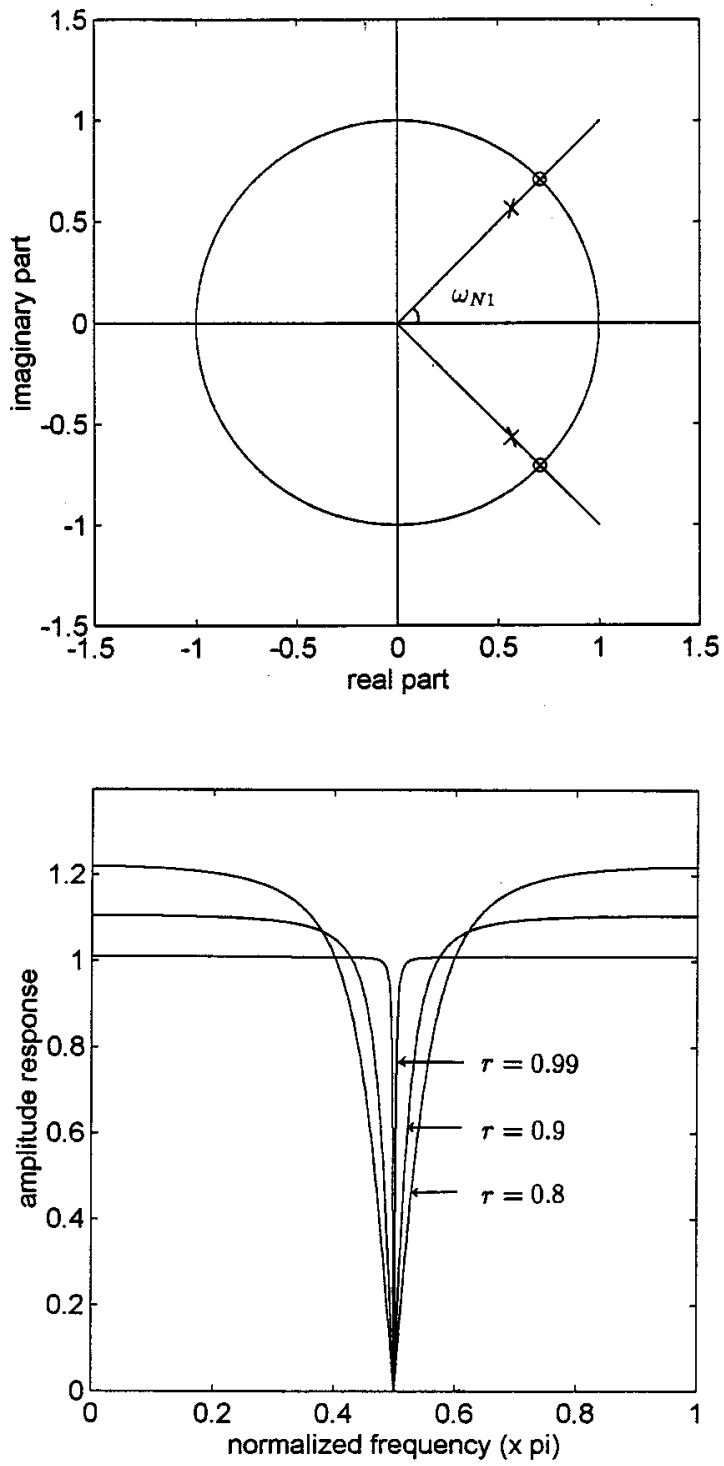

Fig. 1. Type I IIR notch filter. (Top) Pole-zero diagram ( $x$ : pole o: zero). (Bottom) Amplitude response for various pole radius $r$ values.

where $B(z)=\sum_{k=0}^{2 M} b_{k} z^{-k}$ is a symmetrical polynomial, that is

$$
\begin{aligned}
b_{0} & =b_{2 M}=1 \\
b_{2 M-k} & =b_{k} \quad k=1, \ldots, M-1 .
\end{aligned}
$$

Given the notch frequencies $\omega_{N k}(k=1, \ldots, M)$, the coefficients $b_{k}$ can be computed by using a recursive relationship developed in [12]. Moreover, $2 M$ poles and zeros of this filter are given by

zeros : $e^{ \pm j \omega_{N k}}$

poles : $r e^{ \pm j \omega_{N k}} \quad k=1, \ldots, M$.

The zeros are constrained to locate on the unit circle at the notch frequencies $\omega_{N k}$, and the poles are at the same radial lines as zeros. Fig. 1 shows the pole-zero diagram and frequency response for $M=1$ and notch frequency $\omega_{N 1}=0.5 \pi$. For stability, pole radius $r$ must be smaller than one. When $r$ approaches unity, the $H_{1}(z)$ becomes an ideal notch filter.
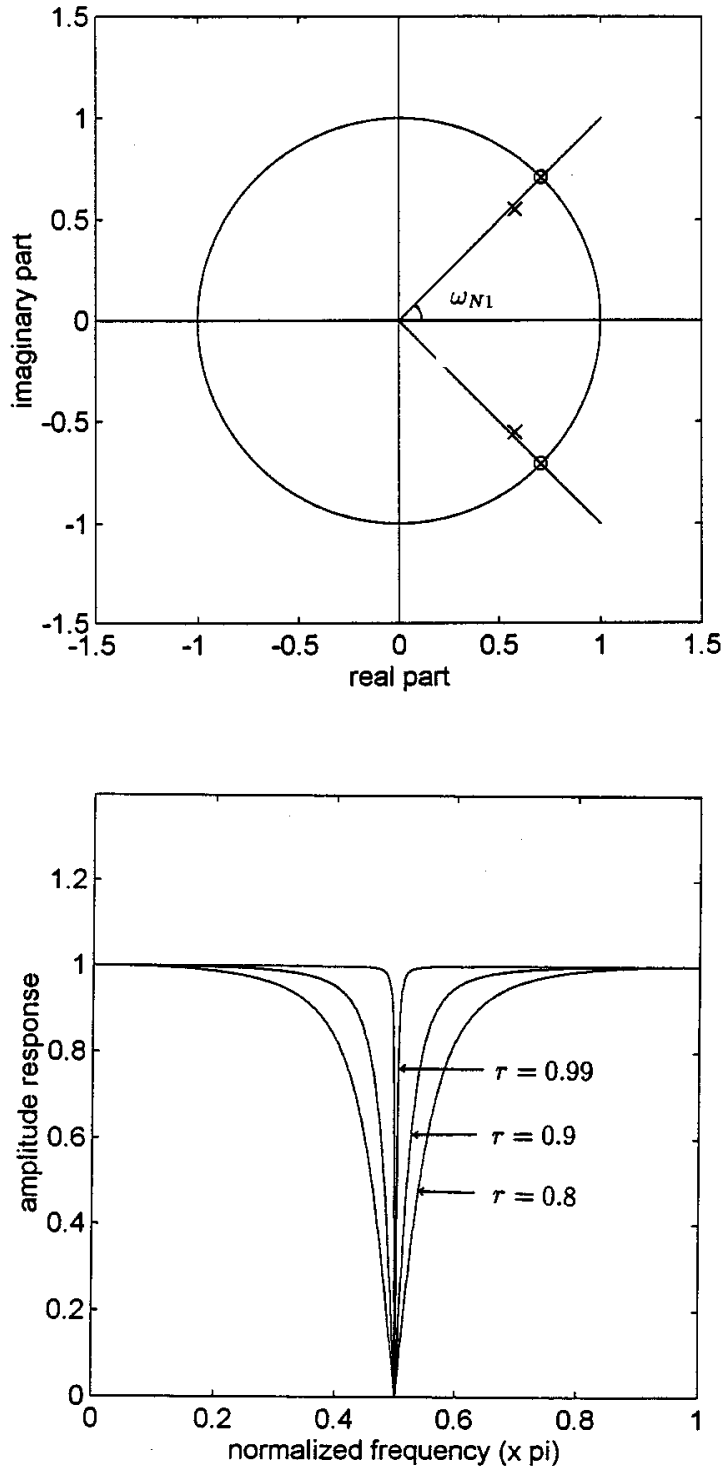

Fig. 2. Type II IIR notch filter. (Top) Pole-zero diagram ( $x$ : pole o: zero). (Bottom) Amplitude response for various pole radius $r$ values.

\section{B. Type II}

The transfer function of this type IIR notch filter has the following form:

$$
H_{2}(z)=\frac{1}{2}(1+F(z))
$$

where $F(z)$ is a $2 M$-order allpass filter given by

$$
F(z)=\frac{f_{2 M}+\cdots+f_{1} z^{-2 M+1}+z^{-2 M}}{1+f_{1} z^{-1}+\cdots+f_{2 M} z^{-2 M}} .
$$

When notch frequencies $\omega_{N k}$ are specified, a procedure to determine the coefficients $f_{k}(k=1, \ldots, 2 M)$ is presented in [13]. The $2 M$ zeros of $H_{2}(z)$ are also at $e^{ \pm \omega_{N k}}$ to make the filter a zero gain at notch frequencies. The $2 M$ poles of $H_{2}(z)$ are all adjacent to the zeros to compensate for the frequency response to be the unit gain for the non-notch band. Fig. 2 shows the pole-zero diagram and frequency response for $M=1$, notch 
frequency $\omega_{N 1}=0.5 \pi$, and allpass filter

$$
F(z)=\frac{r^{2}-\left(1+r^{2}\right) \cos \left(\omega_{N 1}\right) z^{-1}+z^{-2}}{1-\left(1+r^{2}\right) \cos \left(\omega_{N 1}\right) z^{-1}+r^{2} z^{-2}} .
$$

It can be shown that the gains at $\mathrm{dc}$ and Nyquist frequencies are always equal to unity. When $r$ approaches one, the $H_{2}(z)$ becomes an ideal notch filter.

Based on the above description, a two-stage approach to design the notch filter is as follows.

Step 1) Place $2 M$ zeros on the unit circle with angles $\pm \omega_{N k}(k=1, \ldots, M)$. Thus, the numerator of transfer function is equal to $\prod_{k=1}^{M}$ $\left(1-2 \cos \left(\omega_{N k}\right) z^{-1}+z^{-2}\right)$.

Step 2) Place $2 M$ poles inside the unit circle and near $2 M$ zeros to make the frequency response of the non-notch band be the unit gain. When $2 M$ poles approach $2 M$ zeros, the notch filter becomes the ideal one.

From the above discussion, a question may be asked. What is the optimal pole placement? The pole placements in the type I and II notch filters are just two subjective choices. In this paper, we will use the weighted least squares method to find the optimal pole locations. Because the stability of single notch filter is easy to control, let us start with the design of single notch filter in Section III.

\section{Single Notch FILTER DESIGN}

\section{A. Design Method}

The general form of transfer function of single notch filter is expressed as

$$
H(z)=\frac{B(z)}{A(z)}
$$

where the numerator and denominator are given by

$$
\begin{aligned}
& B(z)=1-2 \cos \left(\omega_{N 1}\right) z^{-1}+z^{-2} \\
& A(z)=1-2 r a z^{-1}+r^{2} z^{-2} .
\end{aligned}
$$

Let $a=\cos \left(\omega_{p}\right)$; then, the poles of this filter are $r e^{ \pm j \omega_{p}}$, and the parameter $a$ must satisfy the following linear constraint

$$
-1 \leq a \leq 1
$$

In our design, the pole radius $r$ is specified in advance. Thus, the problem reduces to find the angle $\omega_{p}=\arccos (a)$ such that the cost function

$$
J(a)=\int_{R} W(\omega)\left|1-H\left(e^{j \omega}\right)\right|^{2} d \omega
$$

is minimized, where integral region $R=\left[0, \omega_{N 1}-\epsilon\right] \bigcup\left[\omega_{N 1}+\right.$ $\epsilon, \pi]$ and $W(\omega)$ is a weighting function. Note that $\epsilon$ is a prescribed small positive number. Substituting (6) into (8), we have

$$
\begin{aligned}
J(a) & =\int_{R} W(\omega)\left|1-\frac{B\left(e^{j \omega}\right)}{A\left(e^{j \omega}\right)}\right|^{2} d \omega \\
& =\int_{R} \frac{W(\omega)}{\left|A\left(e^{j \omega}\right)\right|^{2}}\left|A\left(e^{j \omega}\right)-B\left(e^{j \omega}\right)\right|^{2} d \omega \\
& =\int_{R} \frac{W(\omega)}{\left|A\left(e^{j \omega}\right)\right|^{2}}|p(\omega)+q(\omega) a|^{2} d \omega
\end{aligned}
$$

where $p(\omega)$ and $q(\omega)$ are given by

$$
\begin{aligned}
& p(\omega)=\left(r^{2}-1\right) e^{-2 j \omega}+2 \cos \left(\omega_{N 1}\right) e^{-j \omega} \\
& q(\omega)=-2 r e^{-j \omega} .
\end{aligned}
$$

Using the technique described in [19], the optimization problem in (9) can be solved by the following iterative scheme:

$$
\begin{aligned}
J_{k}\left(a_{k}\right) & =\int_{R} \frac{W(\omega)}{\left|A_{k-1}\left(e^{j \omega}\right)\right|^{2}}\left|p(\omega)+q(\omega) a_{k}\right|^{2} d \omega \\
& =\rho_{k-1} a_{k}^{2}+2 \beta_{k-1} a_{k}+\alpha_{k-1}
\end{aligned}
$$

where $\rho_{k-1}, \beta_{k-1}$, and $\alpha_{k-1}$ are

$$
\begin{aligned}
\rho_{k-1} & =\int_{R} \frac{W(\omega)}{\left|A_{k-1}\left(e^{j \omega}\right)\right|^{2}}|q(\omega)|^{2} d \omega \\
\beta_{k-1} & =\int_{R} \frac{W(\omega)}{\left|A_{k-1}\left(e^{j \omega}\right)\right|^{2}} \operatorname{Real}\left(p(\omega) q^{*}(\omega)\right) d \omega \\
\alpha_{k-1} & =\int_{R} \frac{W(\omega)}{\left|A_{k-1}\left(e^{j \omega}\right)\right|^{2}}|p(\omega)|^{2} d \omega
\end{aligned}
$$

The notation Real(.) denotes the real part of complex number, and $\operatorname{Imag}($.$) denotes the imaginary part of complex number.$ Note that the denominator polynomial obtained from the preceding iteration is treated as a part of the weighting function in this scheme. Moreover, at the $k$ th iteration, $A_{k-1}\left(e^{j \omega}\right)$ is known; therefore, the parameter $a_{k}$ can be determined by solving the standard quadratic programming problem

$$
\begin{array}{ll}
\text { Minimize } & \rho_{k-1} a_{k}^{2}+2 \beta_{k-1} a_{k}+\alpha_{k-1} \\
& \text { Subject to } \quad-1 \leq a_{k} \leq 1
\end{array}
$$

Since only single variable is considered in this optimiztion problem and cost function is a parabola with its mouth opened up, the unique closed-form optimal solution is obtained as follows:

$$
a_{k}= \begin{cases}1, & \text { if } \frac{-\beta_{k-1}}{\rho_{k-1}}>1 \\ -1, & \text { if } \frac{-\beta_{k-1}}{\rho_{k-1}}<-1 \\ \frac{-\beta_{k-1}}{\rho_{k-1}}, & \text { if }-1 \leq \frac{-\beta_{k-1}}{\rho_{k-1}} \leq 1\end{cases}
$$

Based on the above description, we propose an iterative quadratic programming algorithm for obtaining the notch filter coefficent $a=\cos \left(\omega_{p}\right)$ as follows.

Step 1) Specifiy the pole radius $r$, notch frequency $\omega_{N 1}, \epsilon$ in the integral region $R$, and the weighting function $W(\omega)$.

Step 2) Given initial parameter $a_{0}$, set $k=1$.

Step 3) Compute the values $\rho_{k-1}, \beta_{k-1}$, and $\alpha_{k-1}$ using (11).

Step 4) Calculate the quadratic programming solution in (13) to obtain the new coefficient $a_{k}$.

Step 5) Terminate the iterative procedure if

$$
\left|a_{k}-a_{k-1}\right| \leq \varepsilon
$$

where $\varepsilon$ is a preset small positive number. Otherwise, set $k=k+1$, and go to step 3 . 

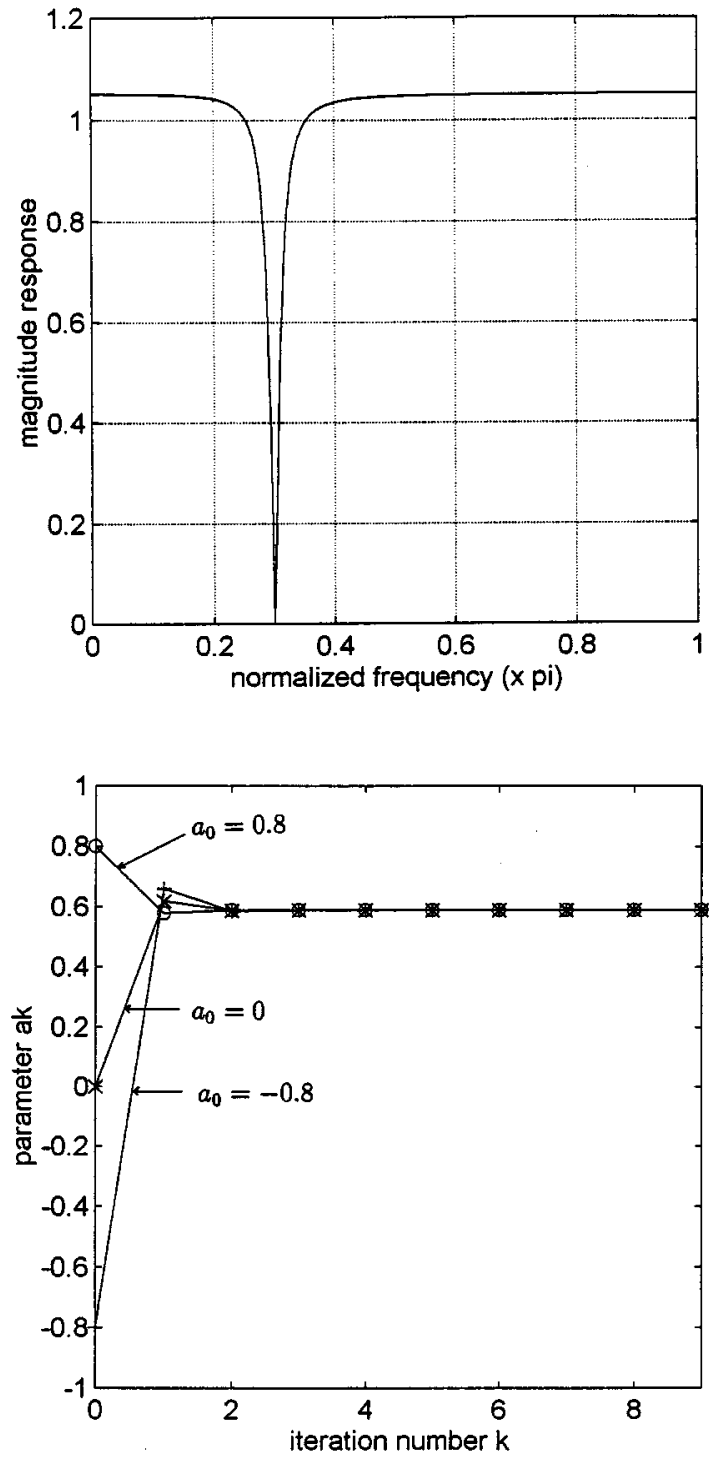

Fig. 3. (Top) Magnitude response of the designed single notch filter. (Bottom) Convergence curves of $a_{k}$ for various initial values $a_{0}$.

\section{B. Design Example}

Here, we present one design example of IIR notch filter to demonstrate the effectiveness of the proposed method. The program is performed in an IBM PC compatible computer (Intel Pentium II CPU insided) using the MATLAB language.

Example 1: In this example, let us consider the following specifications for the notch filter:

- pole radius $r=0.95$;

- notch frequency $\omega_{N 1}=0.3 \pi$;

- $\epsilon=0.001 \pi$,

- weighting function $W(\omega)=1$ for all $\omega$.

The initial parameter $a_{0}$ is chosen as $\cos \left(\omega_{N 1}\right)$. When the proposed algorithm with $\varepsilon=10^{-4}$ is used to design this filter, the resultant magnitude response of this filter is shown in Fig. 3(a). It is clear that the specification is well satisfied. To illustrate the convergence of the algorithm, the values $a_{k}$ are listed in Table I as a function of the number of iterations. It is seen that the errors
TABLE I

PARAMETER $a_{k}$ IN EXAMPLE 1

\begin{tabular}{c|c}
\hline number of iteration & parameter $a_{k}$ \\
\hline 0 & 0.58778525 \\
\hline 1 & 0.58859912 \\
\hline 2 & 0.58855483 \\
\hline 3 & 0.58855724 \\
\hline 4 & 0.58855711 \\
\hline 5 & 0.58855711 \\
\hline 6 & 0.58855711 \\
\hline
\end{tabular}

do not change significantly past three iterations. Thus, convergence speed of proposed algorithm is very fast.

In order to illustrate that the convergence of the proposed algorithm is insensitive to the choice of initial parameter $a_{0}$, the convergence curves of $a_{k}$ for various initial values are shown Fig. 3(b). Fortunately, all of the curves converges the same final values. Thus, the design algorithm is initially independent. In fact, from an optimization-theoretic viewpoint, (12) is a convex quadratic programming problem that always has a unique minimizer. This is why the algorithm always converges to the same minimizer, regardless of the initial point used.

\section{Discussion}

Given notch frequency $\omega_{N 1}$ and pole radius $r$, type I and type II notch filters can be obtained immediately by simple computation, and our method needs to take a lot of time to produce the optimal notch filter. If we care about the design time, we may discard the optimal method and choose heuristic type I or the type II notch filter. Meanwhile, a criterion must be provided to evaluate whether the type I or the type II notch filter is better. One of the methods is to compute the distance between the poles of two types of notch filters and the poles of optimal one because the zeros for the three notch filters are the same. The smaller the distance is, the better the notch filter is. Since three notch filters also have the same pole radius $r$, we only need to compare the pole angles, which are given by

$$
\begin{aligned}
\text { Type I: pole angle } & =\omega_{N 1} \\
\text { Type II: pole angle } & =\arccos \left(\frac{1+r^{2}}{2 r} \cos \left(\omega_{N 1}\right)\right) \\
\text { Optimal: pole angle } & =\arccos (a) .
\end{aligned}
$$

Tables II-IV list three pole angles for various notch frequencies $\omega_{N 1}$, pole radii $r$, and $W(\omega)=1$. It is clear that type II is closer to the optimal one than the type I notch filter. Thus, type II is a better choice than type I in this sense.

\section{Multiple Notch FiLTER Design}

The general form of the transfer function of IIR multiple notch filter is expressed as

$$
H(z)=\frac{B(z)}{A(z)}
$$


TABLE II

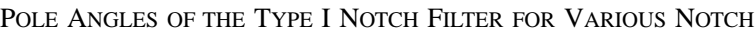
FREQUENCIES $\omega_{N 1}$ AND POLE RADIUS $r$

\begin{tabular}{l|c|c|c|c}
\hline & $r=0.6$ & $r=0.7$ & $r=0.8$ & $r=0.9$ \\
\hline$\omega_{N 1}=0.2 \pi$ & 0.6283 & 0.6283 & 0.6283 & 0.6283 \\
\hline$\omega_{N 1}=0.4 \pi$ & 1.2566 & 1.2566 & 1.2566 & 1.2566 \\
\hline$\omega_{N 1}=0.7 \pi$ & 2.1991 & 2.1991 & 2.1991 & 2.1991 \\
\hline
\end{tabular}

TABLE III

Pole ANGLES OF THE TyPe II NOTCH Filter FOR VARIOUS NOTCH FREQUENCIES $\omega_{N 1}$ AND POLE RADIUS $r$

\begin{tabular}{l|c|c|c|c}
\hline & $r=0.6$ & $r=0.7$ & $r=0.8$ & $r=0.9$ \\
\hline$\omega_{N 1}=0.2 \pi$ & 0.4106 & 0.5335 & 0.5930 & 0.6206 \\
\hline$\omega_{N 1}=0.4 \pi$ & 1.2130 & 1.2357 & 1.2485 & 1.2548 \\
\hline$\omega_{N 1}=0.7 \pi$ & 2.2998 & 2.2467 & 2.2174 & 2.2032 \\
\hline
\end{tabular}

TABLE IV

Pole Angles of the Optimal Notch Filter for Various Notch FREQUENCIES $\omega_{N 1}$ AND POLE RADIUS $r$

\begin{tabular}{l|c|c|c|c}
\hline & $r=0.6$ & $r=0.7$ & $r=0.8$ & $r=0.9$ \\
\hline$\omega_{N 1}=0.2 \pi$ & 0.4104 & 0.5320 & 0.5915 & 0.6194 \\
\hline$\omega_{N 1}=0.4 \pi$ & 1.2129 & 1.2354 & 1.2482 & 1.2545 \\
\hline$\omega_{N 1}=0.7 \pi$ & 2.3000 & 2.2474 & 2.2182 & 2.2038 \\
\hline
\end{tabular}

where the numerator $B(z)$ is defined in (2), and the denominator $A(z)$ is denoted by

$$
A(z)=\prod_{k=1}^{M}\left(1-2 r \cos \left(\omega_{p k}\right) z^{-1}+r^{2} z^{-2}\right)
$$

$r$ is the pole radius, and $\omega_{p k}$ are pole angles. The transfer function $H(z)$ is a generalization of the conventional type I notch filter. When $\omega_{p k}$ are chosen as notch frequencies $\omega_{N k}, H(z)$ becomes the type I notch filter $H_{1}(z)$ in (2). However, this choice is not optimal. The best choice can be obtained by finding the pole angles $\left\{\omega_{p 1}, \omega_{p 2} \ldots, \omega_{p M}\right\}$ to minimize the cost function

$$
J\left(\omega_{p 1}, \ldots, \omega_{p M}\right)=\int_{R} W(\omega)\left|1-H\left(e^{j \omega}\right)\right|^{2} d \omega .
$$

Because $J$ is not a quadratic function of angles $\omega_{p k}$, the quadratic programming approach cannot be applied to solve this nonlinear optimization problem. In order to obtain a satisfactory solution, we rewrite $A(z)$ in (16) as follows:

$$
A(z)=\sum_{k=0}^{2 M} a_{k} r^{k} z^{-k} .
$$

The coefficients of $A(z)$ has the following symmetrical property: $a_{0}=a_{2 M}=1$ and $a_{2 M-k}=a_{k}(k=1, \ldots, M-1)$.
Define two vectors $\mathbf{a}=\left[a_{1}, \ldots, a_{M}\right]^{t}$ and

$$
\mathbf{e}(z)=\left[\begin{array}{c}
r z^{-1}+r^{2 M-1} z^{-(2 M-1)} \\
r^{2} z^{-2}+r^{2 M-2} z^{-(2 M-2)} \\
\vdots \\
r^{M-1} z^{-(M-1)}+r^{M+1} z^{-(M+1)} \\
r^{M} z^{-M}
\end{array}\right] .
$$

Then, the polynomial $A(z)$ can be rewritten as

$$
A(z)=1+r^{2 M} z^{-2 M}+\mathbf{a}^{t} \mathbf{e}(z) .
$$

Instead of finding pole angles $\omega_{p k}$ to minimize function $J$, we will find coefficient vector a to minimize $J$. Thus, (17) can be rewritten as

$$
\begin{aligned}
J(\mathbf{a}) & =\int_{R} W(\omega)\left|1-H\left(e^{j \omega}\right)\right|^{2} d \omega \\
& =\int_{R} W(\omega)\left|1-\frac{B\left(e^{j \omega}\right)}{A\left(e^{j \omega}\right)}\right|^{2} d \omega \\
& =\int_{R} \frac{W(\omega)}{\left|A\left(e^{j \omega}\right)\right|^{2}}\left|u(\omega)+\mathbf{v}(\omega)^{t} \mathbf{a}\right|^{2} d \omega
\end{aligned}
$$

where $u(\omega)$ and $\mathbf{v}(\omega)$ are given by

$$
\begin{aligned}
& u(\omega)=1+r^{2 M} e^{-j 2 M \omega}-\sum_{k=0}^{2 M} b_{k} e^{-j k \omega} \\
& \mathbf{v}(\omega)=\mathbf{e}\left(e^{j \omega}\right)
\end{aligned}
$$

and the integral region $R=\left[0, \omega_{N 1}-\epsilon\right] \bigcup\left[\omega_{N 1}+\epsilon, \omega_{N 2}-\right.$ $\epsilon] \bigcup \cdots \cup\left[\omega_{N M}+\epsilon, \pi\right]$. Using the technique developed in [19], the optimization problem in (21) can be solved by the following iterative scheme:

$$
\begin{aligned}
J_{k}\left(\mathbf{a}_{k}\right) & =\int_{R} \frac{W(\omega)}{\left|A_{k-1}\left(e^{j \omega}\right)\right|^{2}}\left|u(\omega)+\mathbf{v}(\omega)^{t} \mathbf{a}_{k}\right|^{2} d \omega \\
& =\mathbf{a}_{k}^{t} \mathbf{Q}_{k-1} \mathbf{a}_{k}+2 \mathbf{p}_{k-1}^{t} \mathbf{a}_{k}+c_{k-1}
\end{aligned}
$$

where matrix $\mathbf{Q}_{k-1}$, vectors $\mathbf{p}_{k-1}$, and scalar $c_{k-1}$ are

$$
\begin{aligned}
c_{k-1} & =\int_{R} \frac{W(\omega)}{\left|A_{k-1}\left(e^{j \omega}\right)\right|^{2}}|u(\omega)|^{2} d \omega \\
\mathbf{p}_{k-1} & =\int_{R} \frac{W(\omega)}{\left|A_{k-1}\left(e^{j \omega}\right)\right|^{2}} \operatorname{Real}\left(\mathbf{v}(\omega) u^{*}(\omega)\right) d \omega \\
\mathbf{Q}_{k-1} & =\int_{R} \frac{W(\omega)}{\left|A_{k-1}\left(e^{j \omega}\right)\right|^{2}} \operatorname{Real}\left(\mathbf{v}(\omega) \mathbf{v}^{H}(\omega)\right) d \omega .
\end{aligned}
$$

Note that the denominator polynomial obtained from the preceding iteration is treated as part of the weighting function in this scheme. Moreover, at the $k$ th iteration, $A_{k-1}(\omega)$ is known; therefore, the parameter $\mathbf{a}_{k}$ can be determined by solving the following optimization problem:

$$
\begin{array}{cl}
\text { Minimize } & \mathbf{a}_{k}^{t} \mathbf{Q}_{k-1} \mathbf{a}_{k}+2 \mathbf{p}_{k-1}^{t} \mathbf{a}_{k}+c_{k-1} \\
\text { Subject to } & \text { the zeros of } A_{k}(z) \text { are all inside } \\
& \text { the unit circle. }
\end{array}
$$

Because the symmetry of the coefficients $a_{k}$ is only necessary but not sufficient for all poles to lie on a circle with radius $r$, the 
condition $0 \leq r<1$ does not guarantee that the filter is stable as in the single notch filter case. Thus, the stability problem must be solved by imposing a set of linear constraints on the coefficient $\mathbf{a}_{k}$ of $A_{k}(z)$ such that all of zeros of $A_{k}(z)$ are inside the unit circle. Fortunately, Lang has proposed an interesting method to solve this problem [14]. This method is mainly based on Rouché's theorem.

Rouché's Theorem: If $f(z)$ and $g(z)$ are analytic inside and on a closed contour $C$, and $|g(z)|<|f(z)|$ on $C$, then $f(z)$ and $f(z)+g(z)$ have the same number of zeros inside $C$.

A proof of Rouché's theorem can be found in [15]. Now, let the contour $C$ be the unit circle, and let the denominator polynomial $A_{k-1}(z)$ at iteration step $k-1$ have all its zeros inside the unit circle; then, according to Rouché's theorem, the denominator polynomial in the next iteration $k$ is given by

$$
A_{k}(z)=A_{k-1}(z)+\alpha \Delta_{k}(z) \quad 0<\alpha<1
$$

and will also have all its zeros inside unit circle if the following condition is satisfied:

$$
\left|\Delta_{k}(z)\right| \leq\left|A_{k-1}(z)\right| \quad|z|=1
$$

Since two polynomials $A_{k}(z)$ and $\Delta_{k}(z)$ are given by

$$
\begin{aligned}
& \Delta_{k}(z)=\mathbf{d}_{k}^{t} \mathbf{e}(z) \\
& A_{k}(z)=1+r^{2 M} z^{-2 M}+\mathbf{a}_{k}^{t} \mathbf{e}(z)
\end{aligned}
$$

where $\mathbf{e}(z)$ has been defined in (19) and

$$
\begin{aligned}
\mathbf{a}_{k} & =\left[a_{1}, a_{2}, \ldots, a_{M}\right]_{k}^{t} \\
\mathbf{d}_{k} & =\left[\delta_{1}, \delta_{2}, \ldots, \delta_{M}\right]_{k}^{t}
\end{aligned}
$$

(25) can be rewritten as

$$
\mathbf{a}_{k}=\mathbf{a}_{k-1}+\alpha \mathbf{d}_{k} .
$$

Substituting (28) into (22), we obtain

$$
\begin{aligned}
J_{k}\left(\mathbf{a}_{k}\right)= & \mathbf{a}_{k}^{t} \mathbf{Q}_{k-1} \mathbf{a}_{k}+2 \mathbf{p}_{k-1}^{t} \mathbf{a}_{k}+c_{k-1} \\
= & \left(\mathbf{a}_{k-1}+\alpha \mathbf{d}_{k}\right)^{t} \mathbf{Q}_{k-1}\left(\mathbf{a}_{k-1}+\alpha \mathbf{d}_{k}\right) \\
& +2 \mathbf{p}_{k-1}^{t}\left(\mathbf{a}_{k-1}+\alpha \mathbf{d}_{k}\right)+c_{k-1} \\
= & \mathbf{d}_{k}^{t} \overline{\mathbf{Q}}_{k-1} \mathbf{d}_{k}+2 \overline{\mathbf{p}}_{k-1}^{t} \mathbf{d}_{k}+\bar{c}_{k-1}
\end{aligned}
$$

where

$$
\begin{aligned}
\overline{\mathbf{Q}}_{k-1} & =\alpha^{2} \mathbf{Q}_{k-1} \\
\overline{\mathbf{p}}_{k-1} & =\alpha \mathbf{Q}_{k-1} \mathbf{a}_{k-1}+\alpha \mathbf{p}_{k-1} \\
\bar{c}_{k-1} & =\mathbf{a}_{k-1}^{t} \mathbf{Q}_{k-1} \mathbf{a}_{k-1}+2 \mathbf{p}_{k-1}^{t} \mathbf{a}_{k-1}+c_{k-1}
\end{aligned}
$$

Next, we study the constraints in (26). Let $\Theta=\left\{\theta_{i} \mid i=\right.$ $1, \ldots, L\}$ be the set of dense grid points on $[0, \pi]$; then, this constraint, on the set $\Theta$, reduces to

$$
\left|\Delta_{k}\left(e^{j \theta_{i}}\right)\right| \leq\left|A_{k-1}\left(e^{j \theta_{i}}\right)\right| \quad i=1, \ldots, L .
$$

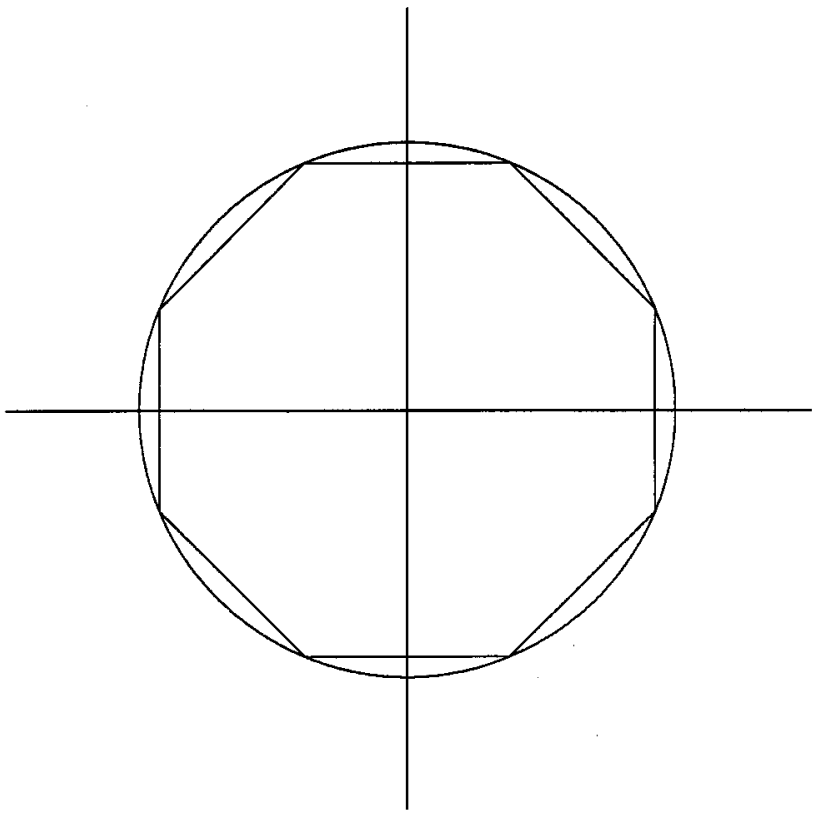

Fig. 4. Approximation of a circle with an octagon.

Letting $g_{i}=\left|A_{k-1}\left(e^{j \theta_{i}}\right)\right|$, the vectors

$$
\begin{aligned}
& \mathbf{r}_{i}=\operatorname{Real}\left(\mathbf{e}\left(e^{j \theta_{i}}\right)\right) \\
& \mathbf{s}_{i}=\operatorname{Imag}\left(\mathbf{e}\left(e^{j \theta_{i}}\right)\right)
\end{aligned}
$$

and two scalars

$$
x_{i}=\mathbf{d}_{k}^{t} \mathbf{r}_{i} \quad y_{i}=\mathbf{d}_{k}^{t} \mathbf{s}_{i}
$$

(31) then becomes

$$
\sqrt{x_{i}^{2}+y_{i}^{2}} \leq g_{i} \quad i=1, \ldots, L
$$

Obviously, it is a quadratic constraint of the coefficients $\mathrm{d}_{k}$. Now, this quadratic constraint can be converted to the linear constraints by approximating a circle with an octagon shown in Fig. 4. This approximation technique has also been used in the design of FIR filters in the complex domain [16]. Thus, the quadratic constraint in (33) can be approximated by the following linear constraints:

$$
\begin{aligned}
\left|\cos \left(\frac{n \pi}{4}\right) x_{i}+\sin \left(\frac{n \pi}{4}\right) y_{i}\right| & \leq g_{i} \cos \left(\frac{\pi}{8}\right) \\
n & =0,1,2,3 \\
i & =1, \ldots, L .
\end{aligned}
$$

It is clear that if $\left(x_{i}, y_{i}\right)$ satisfies the linear constraints in (34) simultaneously, the $\left(x_{i}, y_{i}\right)$ must satisfy the quadratic constraint in (33). Substituting (32) into (34), we obtain

$$
\mathbf{T}_{i} \mathbf{d}_{k} \leq \mathbf{h}_{i} \quad i=1, \ldots, L
$$


where matrix $\mathbf{T}_{i}$ and vector $\mathbf{h}_{i}$ are given by

$$
\mathbf{T}_{i}=\left[\begin{array}{c}
\mathbf{r}_{i}^{t} \\
-\mathbf{r}_{i}^{t} \\
\left(\frac{\mathbf{r}_{i}+\mathbf{s}_{i}}{\sqrt{2}}\right)^{t} \\
-\left(\frac{\mathbf{r}_{i}+\mathbf{s}_{i}}{\sqrt{2}}\right)^{t} \\
\mathbf{s}_{i}^{t} \\
-\mathbf{s}_{i}^{t} \\
\left(\frac{-\mathbf{r}_{i}+\mathbf{s}_{i}}{\sqrt{2}}\right)^{t} \\
-\left(\frac{-\mathbf{r}_{i}+\mathbf{s}_{i}}{\sqrt{2}}\right)^{t}
\end{array}\right] \quad \mathbf{h}_{i}=g_{i} \cos \left(\frac{\pi}{8}\right)\left[\begin{array}{l}
1 \\
1 \\
1 \\
1 \\
1 \\
1 \\
1 \\
1
\end{array}\right]
$$

Using matrix notation, the condition in (35) becomes

$$
\mathbf{T d}_{k} \leq \mathbf{h}
$$

where

$$
\mathbf{T}=\left[\begin{array}{c}
\mathbf{T}_{1} \\
\mathbf{T}_{2} \\
\vdots \\
\mathbf{T}_{L}
\end{array}\right]_{8 L \times 2 M} \mathbf{h}=\left[\begin{array}{c}
\mathbf{h}_{1} \\
\mathbf{h}_{2} \\
\vdots \\
\mathbf{h}_{L}
\end{array}\right]_{8 L \times 1}
$$

Thus, the problem in (24) can be rewritten as the standard quadratic programming problem as

$$
\begin{gathered}
\text { Minimize } \quad \mathbf{d}_{k}^{t} \overline{\mathbf{Q}}_{k-1} \mathbf{d}_{k}+2 \overline{\mathbf{p}}_{k-1}^{t} \mathbf{d}_{k}+\bar{c}_{k-1} \\
\text { Subject to } \quad \mathbf{T d}_{k} \leq \mathbf{h} .
\end{gathered}
$$

Based on the above description, we propose an iterative quadratic programming algorithm for obtaining the denominator polynomial $A(z)$ of notch filter as follows.

Step 1) Specify the notch frequencies $\omega_{N k}(k=1,2$, $\ldots, M), \epsilon$ in the integral region $R$, weighting function $W(\omega)$, number of grid points $L$, pole radius $r$, and parameter $\alpha$.

Step 2) Specify the initial polynomial $A_{0}(z)=\prod_{k=1}^{M}(1-$ $\left.2 r \cos \left(\omega_{N k}\right) z^{-1}+r^{2} z^{-2}\right)$. Compute the vector $\mathbf{a}_{0}$, and set $k=1$.

Step 3) Use (23) to compute $\mathbf{Q}_{k-1}, \mathbf{p}_{k-1}$, and $c_{k-1}$.

Step 4) Use (30) to compute $\overline{\mathbf{Q}}_{k-1}, \overline{\mathbf{p}}_{k-1}$, and $\bar{c}_{k-1}$.

Step 5) Use (36)-(38) to compute the stability constraint parameters $\mathbf{T}$ and $\mathbf{h}$.

Step 6) Solve the quadratic programming problem in (39) to obtain the coefficient $\mathbf{d}_{k}$.

Step 7) Compute $\mathbf{a}_{k}$ by using (28), i.e.,

$$
\mathbf{a}_{k}=\mathbf{a}_{k-1}+\alpha \mathbf{d}_{k} .
$$

Step 8) Terminate the iterative procedure if

$$
\left|\mathbf{a}_{k}-\mathbf{a}_{k-1}\right| \leq \varepsilon
$$

where $\varepsilon$ is a preset positive number. Otherwise, set $k=k+1$, and go to Step 3 .

The proposed design algorithm for the multiple IIR notch filter depends on the existence of efficient algorithms to solve the quadratic programming (QP) problems that have been known for a long time. In the context of FIR filter design, several algorithms for solving the constrained least squares problem have been presented [17], [18]. On the other hand, using the QP to design IIR filter has also been investigated recently [19], [20]. In this paper, the subroutine QP.m in the optimization toolbox of MATLAB software is used to solve QP problem.

In the following, we present two design examples of the IIR multiple notch filter to illustrate the effectiveness of the proposed method. One is where distances between notch frequencies are large, and the other is where distances are small.

Example 2: In this example, let us consider the following design parameters for the IIR multiple notch filter:

- notch frequencies $\omega_{N 1}=0.1 \pi, \omega_{N 2}=0.3 \pi, \omega_{N 3}=$ $0.5 \pi, \omega_{N 4}=0.8 \pi$

- pole radius $r=0.95, \epsilon=0.001 \pi, \alpha=0.99, L=200$, $\varepsilon=10^{-4}$;

- weighting function $W(\omega)=1$ for all $\omega$.

The design algorithm converges after three iterations. The resultant amplitude response of this filter and pole-zero diagram is shown in Fig. 5. It is clear that the specification is well satisfied. Moreover, the square error defined in (21) is 0.5982 for the designed filter, 0.5989 for the conventional type II notch filter, and 0.6002 for the conventional type I notch filter. Thus, our approach provides a smaller design error than the conventional methods. Eight poles of the designed notch filter are given in the first expression shown at the bottom of the next page. Clearly, the radii of all poles are equal to the prescribed value 0.95 ; hence, the filter is stable. From this result, we see that the symmetric condition can make poles lie on a circle with prescribed radius $r$ if notch frequencies are well separate.

Example 3: The design parameters of this example are same as those of Example 2, except that the notch frequencies $\omega_{N 1}=0.1 \pi, \omega_{N 2}=0.2 \pi, \omega_{N 3}=0.5 \pi$, and $\omega_{N 4}=0.8 \pi$, and the pole radius $r=0.8$. That is, we let the notch frequency $\omega_{N 1}$ be very close to $\omega_{N 2}$. The design algorithm converges after 12 iterations. The resultant amplitude response and pole-zero diagrams are shown in Fig. 6. From this result, we see that the amplitude response is almost equiripple in the non-notch band. The squares error defined in (21) is 2.2029 for the designed filter, 2.3246 for conventional type II notch filter, and 2.8750 for the conventional type I notch filter. Thus, our approach provides smaller design error than the conventional methods. Moreover, eight poles of the designed notch filter are given by the second expression at the bottom of the next page. Clearly, the maximum pole radius is equal to 0.9104; hence, the designed filter is stable. From this result, we see that the symmetric condition can make poles corresponding to well-separate notch frequencies $0.8 \pi$ and $0.5 \pi$ be on a circle with prescribed radius $r=0.8$, but it cannot make poles corresponding to close notch frequencies $0.1 \pi$ and $0.2 \pi$ be on the prescribed circle. This result is because that symmetric condition is only necessary but is not a sufficient condition. Rouché's theorem is only used to make those poles corresponding to close notch frequencies be inside the unit circle to guarantee that the filter is stable. 

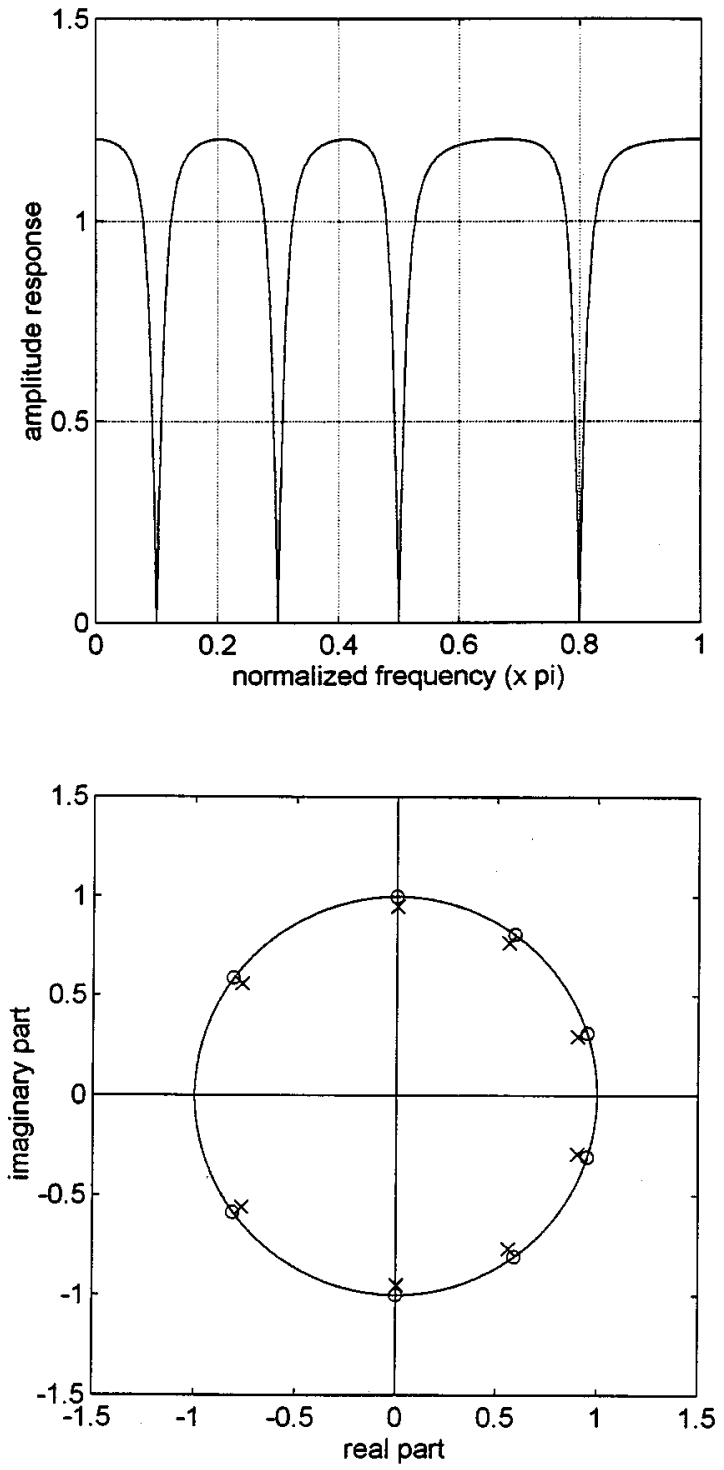

Fig. 5. Design results in the Example 2. (Top) Amplitude response. (Bottom) Pole-zero diagram ( $x$ : pole o:zero).

\section{CONCLusions}

In this paper, a two-stage approach to design stable IIR notch filters has been presented. First, the numerator of the transfer function of the IIR notch filter is obtained by placing the zeros on the unit circle with angles equal to the prescribed notch frequencies. Then, the denominator polynomial is determined by using an iterative scheme in which the optimal pole placements are found by solving a standard quadratic programming
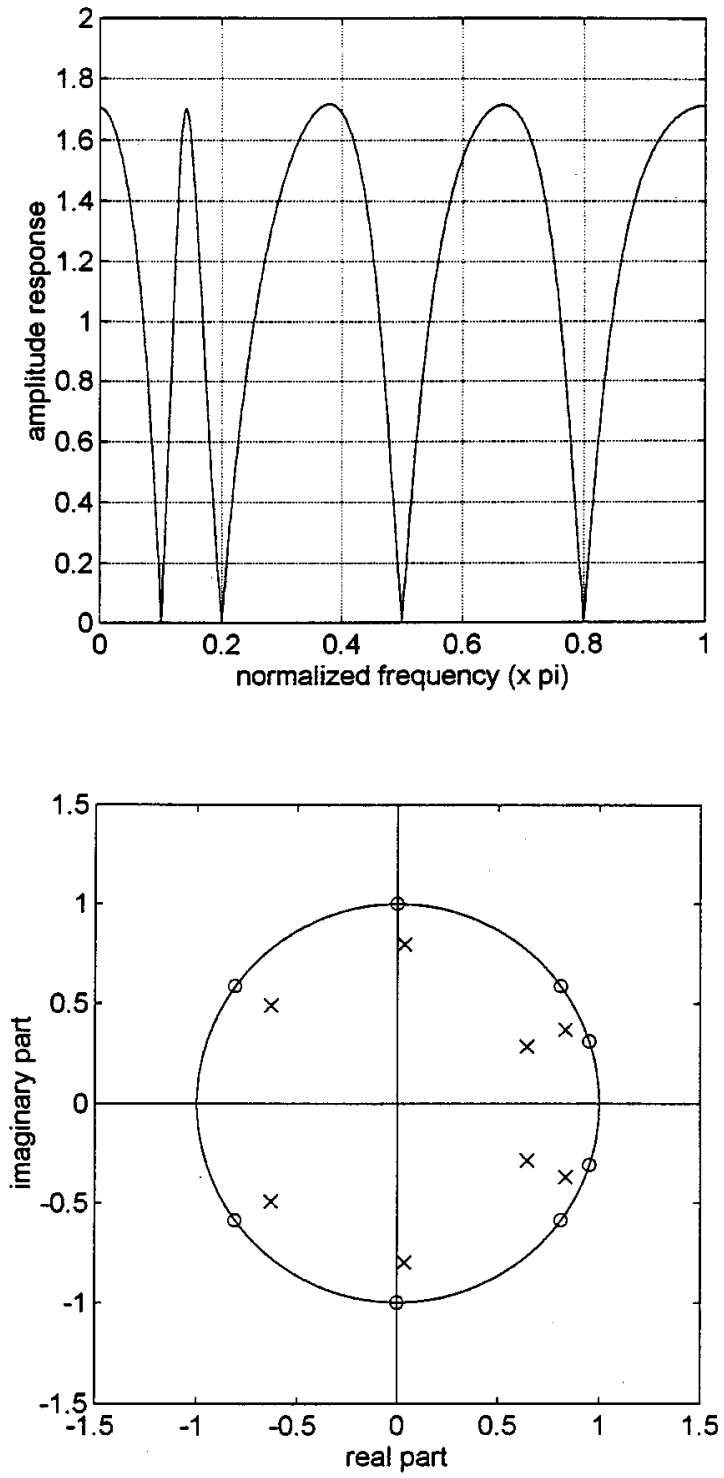

Fig. 6. Design results in Example 3. (Top) amplitude response. (Bottom) pole-zero diagram ( $x$ : pole o:zero).

problem. For stability, the pole radius in the single notch filter design is specified by the designer, and in the multiple notch filter design, the pole radius is constrained by using the implications of Rouché's theorem. Examples are included to illustrate the effectiveness of the proposed technique. However, only 1-D notch filters are considered here. Thus, it is interesting to extend this method to the design of 2-D notch filters. This topic will be studied in the future.

$$
0.95 e^{ \pm j 0.7993 \pi} \quad 0.95 e^{ \pm j 0.4987 \pi} \quad 0.95 e^{ \pm j 0.2995 \pi} \quad 0.95 e^{ \pm j 0.0999 \pi}
$$

$$
0.8 e^{ \pm j 0.7891 \pi} \quad 0.8 e^{ \pm j 0.485 \check{5} \pi} \quad 0.9104 e^{ \pm j 0.1336 \pi} \quad 0.7030 e^{ \pm j 0.1336 \pi} .
$$




\section{REFERENCES}

[1] R. Carney, "Design of a digital notch filter with tracking requirements," IEEE Trans. Space Electron. Telem., vol. SET-9, pp. 109-114, Dec. 1963.

[2] K. Hirano, S. Nishimura, and S. K. Mitra, "Design of digital notch filters," IEEE Trans. Circuits Syst., vol. CAS-21, pp. 540-546, July 1974.

[3] M. H. Er, "Designing notch filter with controlled null width," Signal Process., vol. 24, no. 3, pp. 319-329, Sept. 1991.

[4] G. W. Medlin, "A novel design technique for tuneable notch filters," in Proc. Int. Symp. Circuits Syst., New Orleans, LA, May 1990, pp. 471-474.

[5] S. C. D. Roy, S. B. Jain, and B. Kumar, "Design of digital FIR notch filters," Proc. Inst. Elect. Eng.-Vis. Image Signal Process., vol. 141, pp. 334-338, Oct. 1994.

[6] S. B. Jain, B. Kumar, and S. C. D. Roy, "Design of FIR notch filters by using Bernstein polynomials," Int. J. Circ. Theor. Applicat., vol. 25, pp. 135-139, 1997.

[7] T. H. Yu, S. K. Mitra, and H. Babic, "Design of linear phase FIR notch filters," Sadhana, vol. 15, pp. 133-155, Nov. 1990.

[8] S. C. Pei and C. C. Tseng, "Two dimensional IIR digital notch filter design," IEEE Trans. Circuits Syst. II, vol. 41, pp. 227-231, Mar. 1994.

[9] S. C. Pei, W. S. Lu, and C. C. Tseng, "Analytical two dimensional IIR notch filter design using outer product expansion," IEEE Trans. Circuits Syst. II, vol. 44, pp. 765-768, Sept. 1997.

[10] M. V. Dragosevic and S. S. Stankovic, "An adaptive notch filter with improved tracking properties," IEEE Trans. Signal Processing, vol. 43, pp. 2068-2078, Sept. 1995.

[11] S. C. Pei and C. C. Tseng, "Elimination of AC intereference in electrocardiogram using IIR notch filter with transient suppression," IEEE Trans. Biomed. Eng., vol. 42, pp. 1128-1132, Sept. 1995.

[12] T. S. Ng, "Some aspects of an adaptive digital notch filter with constrained poles and zeros," IEEE Trans. Acoust., Speech, Signal Processing, vol. ASSP-35, pp. 158-161, Feb. 1987.

[13] S. C. Pei and C. C. Tseng, "IIR multiple notch filter design based on allpass filter," IEEE Trans. Circuits Syst. II, vol. 44, pp. 133-136, Feb. 1997.

[14] M. C. Lang, "Weighted least-squares IIR filter design with arbitrary magnitude and phase responses and specified stability margin," in Proc. IEEE Symp. Adv. Digital Filtering Signal Process., Victoria, BC, Canada, June 1998, pp. 82-86.

[15] R. V. Churchill and J. W. Brown, Complex Variables and Applications. New York: McGraw-Hill, 1984

[16] X. Chen and T. W. Parks, "Design of FIR filters in the complex domain," IEEE Trans. Acoust., Speech, Signal Processing, vol. ASSP-35, pp. 144-153, Feb. 1987.

[17] M. Lang, I. W. Selesnick, and C. S. Burrus, "Constrained least squares design of 2-D FIR filters," IEEE Trans. Signal Processing, vol. 44, pp. 1234-1241, May 1996.

[18] J. W. Adams and J. L. Sullivan, "Peak-constrained least-squares optimization," IEEE Trans. Signal Processing, vol. 46, pp. 306-321, Feb. 1998.
[19] W. S. Lu, S. C. Pei, and C. C. Tseng, "A weighted least-squares method for the design of stable 1-D and 2-D IIR digital filters," IEEE Trans. Signal Processing, vol. 46, pp. 1-10, Jan. 1998.

[20] J. L. Sullivan and J. W. Adams, "PCLS IIR digital filters with simultaneous frequency response magnitude and group delay specifications," IEEE Trans. Signal Processing, vol. 46, pp. 2853-2861, Nov. 1998.

Chien-Cheng Tseng (S'90-M'95-SM'01) was born in Taipei, Taiwan, R.O.C., in 1965. He received the B.S. degree, with honors, from Tatung Institute of Technology, Taipei, in 1988 and the M.S. and Ph.D. degrees from the National Taiwan University, Taipei, in 1990 and 1995, respectively, all in electrical engineering.

From 1995 to 1997, he was an Associate Research Engineer with Telecommunication Laboratories, Chunghwa Telecom Company, Ltd., Taoyuan, Taiwan. He is currently an Associate Professor with the Department of Computer and Communication Engineering, National Kaohsiung First University of Science and Technology, Kaohsiung, Taiwan. His research interests include digital signal processing, pattern recognition, and quantum computation.

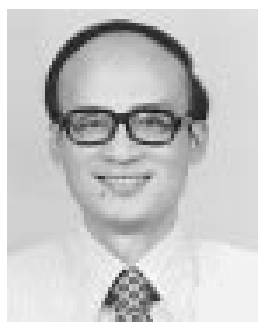

Soo-Chang Pei (S'71-M'86-SM'89-F'00) was born in Soo-Auo, Taiwan, R.O.C. in 1949. He received the B.S.E.E. degree from National Taiwan University (NTU), Taipei, Taiwan, in 1970 and the M.S.E.E. and Ph.D. degrees from the University of California, Santa Barbara, in 1972 and 1975, respectively.

He was an Engineering Officer with the Chinese Navy Shipyard from 1970 to 1971 . From 1971 to 1975, he was a Research Assistant with the University of California, Santa Barbara. He was Professor and Chairman of the Electrical Engineering Department, Tatung Institute of Technology, Taipei, and NTU from 1981 to 1983 and 1995 to 1998, respectively. He is currently a Professor with the Electrical Engineering Department, NTU. His research interests include digital signal processing, image processing, optical information processing, and laser holography.

Dr. Pei received the National Sun Yet-Sen Academic Achievement Award in Engineering in 1984, the Distinguished Research Award from the National Science Council from 1990 to 1998, the Outstanding Electrical Engineering Professor Award from the Chinese Institute of Electrical Engineering in 1998, and the Academic Achievement Award in Engineering from the Ministry of Education in 1998. He was the President of the Chinese Image Processing and Pattern Recognition Society of Taiwan from 1996 to 1998 and is a member of Eta Kappa $\mathrm{Nu}$ and the Optical Society of America. 